\title{
A Forest Pool as a Habitat Island for Mites in a Limestone Forest in Southern Norway
}

\author{
Anna Seniczak ${ }^{1, *}$, Stanisław Seniczak ${ }^{2}$, Radomir Graczyk ${ }^{3}{ }^{\circledR}$, Sławomir Kaczmarek ${ }^{2}$, Bjarte H. Jordal ${ }^{1}$, \\ Jarosław Kowalski ${ }^{4} \mathbb{C}$, Per Djursvoll ${ }^{1}$, Steffen Roth $^{1}$ and Thomas Bolger ${ }^{5,6}$ \\ 1 Department of Natural History, University Museum of Bergen, University of Bergen, P.O. Box 7800, \\ 5020 Bergen, Norway; Bjarte.Jordal@uib.no (B.H.J.); Per.Djursvoll@uib.no (P.D.); Steffen.Roth@uib.no (S.R.) \\ 2 Department Evolutionary Biology, Faculty of Biological Sciences, Kazimierz Wielki University, J.K. \\ Ossolińskich Av. 12, 85-435 Bydgoszcz, Poland; stseni@ukw.edu.pl (S.S.); slawkacz@ukw.edu.pl (S.K.) \\ 3 Department of Biology and Animal Environment, Bydgoszcz University of Science and Technology, \\ Hetmańska 33, 85-039 Bydgoszcz, Poland; graczyk@utp.edu.pl \\ 4 Gorzewo 7, 09-200 Sierpc, Poland; jt.kowalski@o2.pl \\ 5 School of Biology and Environmental Science, University College Dublin, Belfield, \\ D04 V1W8 Dublin, Ireland; tom.bolger@ucd.ie \\ 6 Earth Institute, University College Dublin, Belfield, D04 V1W8 Dublin, Ireland \\ * Correspondence: Anna.Seniczak@uib.no
}

Citation: Seniczak, A.; Seniczak, S.; Graczyk, R.; Kaczmarek, S.; Jordal,

B.H.; Kowalski, J.; Djursvoll, P.; Roth, S.; Bolger, T. A Forest Pool as a Habitat Island for Mites in a Limestone Forest in Southern Norway. Diversity 2021, 13, 578. https://doi.org/10.3390/d13110578

Academic Editor: Tibor Magura

Received: 20 October 2021

Accepted: 9 November 2021

Published: 12 November 2021

Publisher's Note: MDPI stays neutral with regard to jurisdictional claims in published maps and institutional affiliations.

Copyright: (c) 2021 by the authors. Licensee MDPI, Basel, Switzerland. This article is an open access article distributed under the terms and conditions of the Creative Commons Attribution (CC BY) license (https:// creativecommons.org/licenses/by/ $4.0 /)$.

\begin{abstract}
Forest water bodies, e.g., pools, constitute 'environmental islands' within forests, with specific flora and fauna thus contributing considerably to the landscape biodiversity. The mite communities of Oribatida and Mesostigmata in two distinctive microhabitats, water-soaked Sphagnum mosses at the edge of a pool and other mosses growing on the medium-wet forest floor nearby, were compared in a limestone forest in Southern Norway. In total, 16,189 specimens of Oribatida representing 98 species, and 499 specimens of Mesostigmata, from 23 species, were found. The abundance and species number of Oribatida were significantly lower at the pool, while the abundance and species richness of Mesostigmata did not differ. Both the communities of Oribatida and of Mesostigmata differed among the microhabitats studied and analysis showed significant differences between the community structures in the two microhabitats. The most abundant oribatid species in Sphagnum mosses was Parachipteria fanzagoi (Jacot, 1929), which made up over 30\% of all Oribatida, followed by Atropacarus striculus (C.L. Koch, 1835) and Tyrphonothrus maior (Berlese, 1910) (14\% and $12 \%$ of Oribatida, respectively). Among Mesostigmata Paragamasus parrunciger (Bhattacharyya, 1963) dominated (44\% of Mesostigmata), followed by P. lapponicus (Trägårdh, 1910) (14\% of Mesostigmata). Most of these species, except P. lapponicus, were either absent or very uncommon in the other microhabitat studied. The specific acarofauna of the forest pool shows the importance of such microhabitats in increasing forest diversity. In addition, a quarter of the mite species found had not been reported from Norwegian broadleaf forests before, including five new species records for Norway and four new to Fennoscandia, all found in the medium-wet microhabitat. Most of these species are rarely collected and have their northernmost occurrence in the studied forest.
\end{abstract}

Keywords: Oribatida; Mesostigmata; new species records; Norway; Fennoscandia

\section{Introduction}

Forest water bodies, e.g., lakes, ponds, pools or streams, constitute 'environmental islands' within forests, with specific flora and fauna, and are important elements contributing considerably to landscape biodiversity [1,2]. Forest ponds and pools often disappear naturally during the natural succession but in recent years many have disappeared more rapidly due to climatic changes and drainage of large areas for agricultural use [1]. The loss of these water bodies has inestimable effects on entire ecosystems, decreasing water retention [3] and leading to the disappearance of wet habitats that host their unique flora and fauna, including mites and other small invertebrates [4] and included references. 
Forests are very rich in mites. For example, in some Norwegian coniferous forests the density of mites in soil exceeded 1 million individuals per $\mathrm{m}^{2}$ [5], with 48 species of Oribatida and 12 species of Mesostigmata [6]. In broadleaf forests the density is often lower than in coniferous forests (around 50,000 individuals per $\mathrm{m}^{2}$ ), but species richness is greater (approximately $100 \mathrm{spp}$.) [7,8]. Oribatida are usually the dominant mite taxon, and include mainly saprophagous species, which are important in the decomposition processes [9]. The Mesostigmata are a complementary mite group that contains mainly predatory species which control other microarthropod populations in the soil and on the vegetation [9].

Sphagnum spp. can be found in some forests, and they host an abundant and quite diverse mite fauna, especially Oribatida. For example, in one such habitat in Poland, the density of mites varied, depending on the season, from about 50,000 individuals per $\mathrm{m}^{2}$ in winter up to about 90,000 individuals per $\mathrm{m}^{2}$ in autumn, and Oribatida comprised over $90 \%$ of the specimens collected and were represented by 66 species from 30 families [10]. On average, Mesostigmata represented $1.5 \%$ of the individuals but their species composition was not reported.

During a species inventory study of mites in broadleaf forests in Norway we found a forest pool overgrown by Sphagnum mosses in one forest. We hypothesized that this distinct, water-soaked, microhabitat would host different Oribatida and Mesostigmata communities from those in the medium-wet forest floor nearby, thus contributing considerably to the forest biodiversity. In addition, since the limestone forest studied seems very different from other broadleaf forest types with respect to ptyctimous mites [11], we aim to present a more complete picture of the mite fauna based on Oribatida and Mesostigmata found in this forest.

\section{Material and Methods}

\subsection{Study Site}

The limestone forest studied was located in Verpåsen $\left(58.451^{\circ} \mathrm{N} 8.705^{\circ} \mathrm{E}, 62 \mathrm{~m}\right.$ a.s.1.), Arendal municipality, Agder province, Southern Norway (Figure 1). The area is characterized by an oceanic climate, with a mean annual temperature of $7.2{ }^{\circ} \mathrm{C}$ and an annual precipitation of $1010 \mathrm{~mm}$ [12]. The summers are relatively warm with an average temperature of $19.0^{\circ} \mathrm{C}$ in July and August. In the coldest months (January and February) the average temperature is $-1{ }^{\circ} \mathrm{C}$. The vegetation zone is boreonemoral and markedly oceanic [13].

The forest studied has an area of 6.8 ha and is situated on the southeastern Norwegian bedrock area that consists mainly of gneiss with a district direction southwest-northeast, parallel to the coast, but also some granite. It is situated on an amphibolite ridge with heterogeneous terrain (hilly, rock walls, stone blocks, lime rich patches) and a small valley along the stream. The forest is medium wet and dominated by spruce (Picea abies) with occasional oak trees (mostly Quercus robur) and a strong mosaic pattern related to nutritional and lime-richness. Rich parts are otherwise characterized by large hazels (Corylus avellana). The herb layer is species-rich with a total of 16 red-list species recorded. Based on this the site is a High Conservation Value Forest (category A-very important) according to the Norwegian Environment Agency [14]. The area was probably an old pasture forest and may have been more open in the past, probably with more oak and hazel and less spruce [14]. 


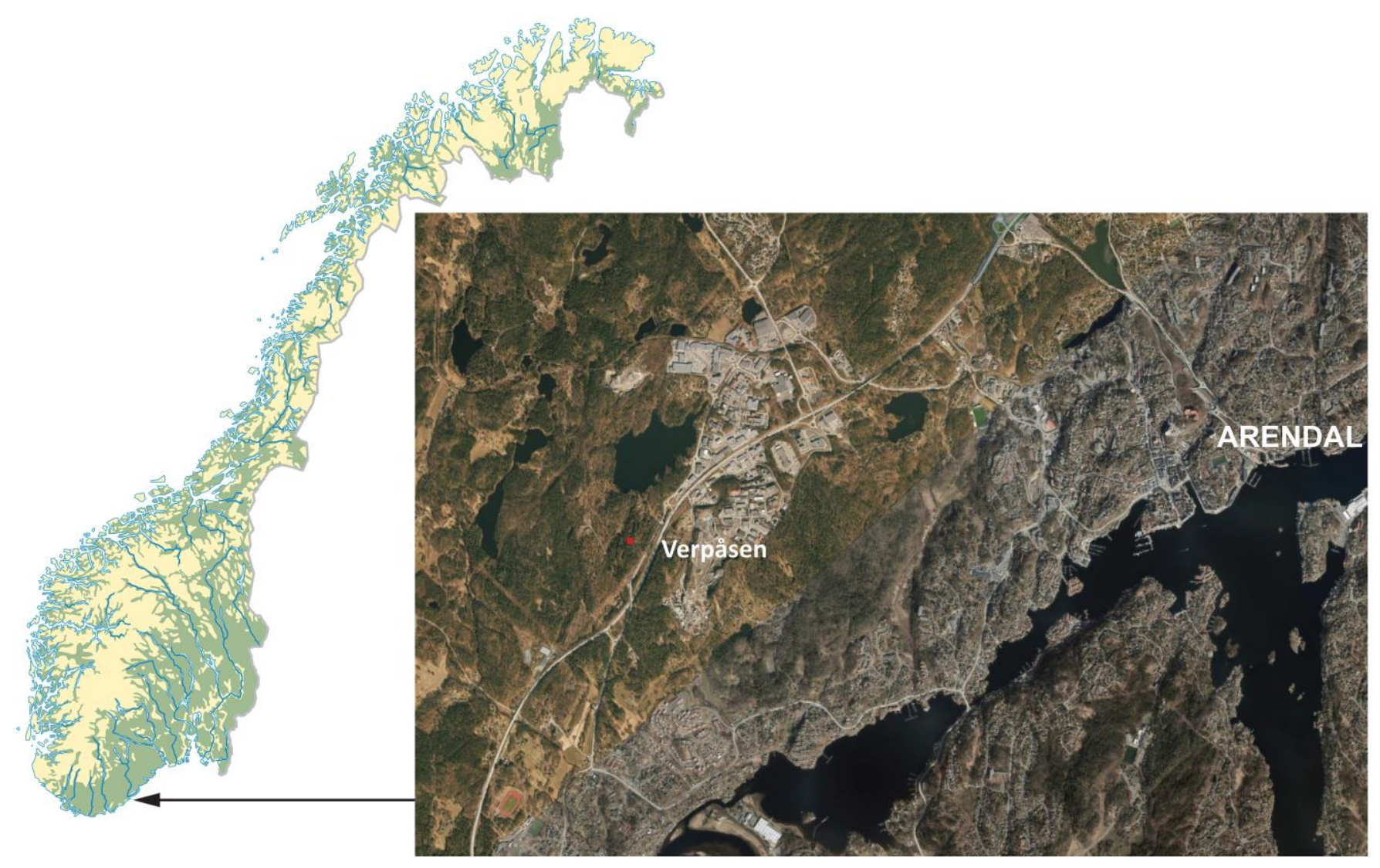

Figure 1. Location in Southern Norway of the limestone forest studied (modified from https:/ / www.norgeskart.no (accessed on 8 June 2021)).

\subsection{Sampling and Identification}

In total, 11 samples, each with a volume of $500 \mathrm{~cm}^{3}$ (ca $100 \mathrm{~cm}^{2}$ in area and $5 \mathrm{~cm}$ deep), were collected by hand on 12 June 2017 from two types of forest microhabitat (Figure 2), (A) water-soaked Sphagnum mosses at the edge of a pool (five samples) and, (B) other mosses growing on the medium-wet forest floor nearby (six samples). Mites were extracted using modified Tullgren funnels for 14 days into $90 \%$ ethanol and sorted out from the samples under stereomicroscope. Oribatida were mounted on cavity slides in $90 \%$ lactic acid (AnalaR NORMAPUR, VWR Chemicals, Belgium) and adult specimens were identified using the keys [15-18], while juveniles were identified based on other publications [19-38]. The nomenclature of oribatid species follows [39-41] and partly [18,33,34]. Mesostigmata were mounted on permanent slides in PVA mounting medium (lactic acid, poly vinyl acetate and phenol solution, BioQuip Products, Inc., Compton, CA, USA) and identified following [42-51]. Information on other mite groups that were sorted out from the samples will be published later. Full names of species are given in Table 1. The arrangement of genera within families and the arrangement of species within genera is alphabetical, except in Table 2, where the species are ordered according to their preferences to the microhabitat. All species are deposited in 90\% ethanol at the University Museum of Bergen, Norway (ZMBN).

The new records of Oribatida for Norway are based on the checklist [52] and later publications [7] and included references, [8,11,53-60]. Those new to Fennoscandia are based on [61-76]. The new records of Mesostigmata for Norway are based on [77-82] and those new to Fennoscandia are based on $[83,84]$. 


\subsection{Statistical Analyses}

Oribatid and mesostigmatid mite populations were characterized by the abundance ( $A$ in $\left.500 \mathrm{~cm}^{3}\right)$, dominance $(D$, percentage of a particular species among Oribatida) and constancy $(C$, percentage of the samples where the species was present) indices, and their communities were characterized by the number of species $(S)$ and the Shannon $\left(H^{\prime}\right)$ diversity index [85]. The basic statistical descriptors included the mean values and standard deviation. Equality of variance was tested with the Levene test, and normality of the distribution was tested with the Kolmogorov-Smirnov test. As the assumptions of variance analysis were not met, non-parametric tests were employed. ANOVA rank KruskalWallis was utilized to test for significant differences between means [86]. Detrended correspondence analysis (DCA) was used to find the main gradients of Oribatida and Mesostigmata communities [87,88]. In scatter plot, the 'joint plot' function was used to visualize better the distribution of the data. These statistics were computed using STATISTICA 13.3 [89], MVSP 3.2 [90] and MS Excel 365 software [91].

A multivariate statistical test, PERMANOVA, was used to assess the significance of differences among locations for the Oribatida and Mesostigmata. This was carried out on Hellinger transformed data using Euclidean distance as the distance index. The tests were carried out using the 'adonis' function in the 'vegan' package in R with 999 permutations [92].

Indicator species analysis was used to identify species which showed a preference for one or other of the microhabitats. Two components are reported: 'specificy' which is the probability that a sample comes from the particular microhabitat if that species is present and 'fidelity' which is the probability of finding the species in a sample from a particular microhabitat [93,94]. The analysis was carried out using 'indicspecies' package in $\mathrm{R}$ [94]. The significance level for all analysis was accepted $\alpha=0.05$.

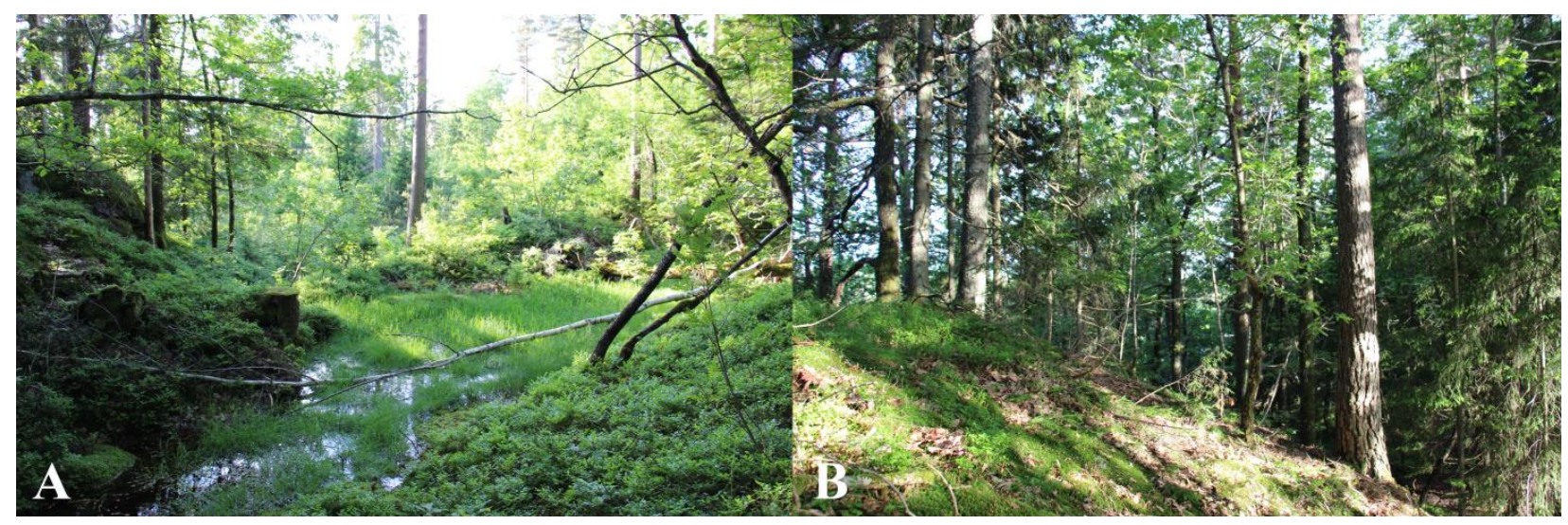

Figure 2. Studied microhabitats: (A) water-soaked Sphagnum on pool and, (B) other mosses on medium-wet forest floor nearby in a limestone forest in Southern Norway.

\section{Results}

In total, 16,189 specimens of Oribatida representing 98 species and 34 families, and 499 specimens of Mesostigmata, from 23 species and nine families were found in the present study (Table 1). Oribatida were less abundant and less diverse in Sphagnum on pools than in other mosses growing on medium-wet forest floor nearby while Mesostigmata had similar abundance and species richness in both types of studied microhabitats (Figure 3). 
Table 1. Oribatida and Mesostigmata in two microhabitats: A-Sphagnum on pool and, B-other mosses on medium-wet forest floor nearby in a limestone forest in Southern Norway; $A$-average abundance in $500 \mathrm{~cm}^{3}, C$-constancy index, $D$-dominance index; abbreviations for detrended correspondence analysis (DCA) for species with $D \geq 1.0$; in bold-new record for Norway; underlined—new record for Fennoscandia; ns—not significant.

\begin{tabular}{|c|c|c|c|c|c|c|c|c|c|c|}
\hline \multirow{2}{*}{$\begin{array}{c}\begin{array}{c}\text { Order/Suborder } \\
\text { and Family }\end{array} \\
\text { Oribatida }\end{array}$} & \multirow[t]{2}{*}{ Species } & \multirow[t]{2}{*}{$\begin{array}{l}\text { Abbreviations } \\
\text { for DCA } \\
\text { Analysis }\end{array}$} & \multicolumn{3}{|c|}{$\begin{array}{c}\text { Microhabitat } \\
\text { A }\end{array}$} & \multicolumn{3}{|c|}{$\begin{array}{l}\text { Microhabitat } \\
\text { B }\end{array}$} & \multicolumn{2}{|c|}{$\begin{array}{l}\text { ANOVA Rang } \\
\text { Kruskal-Wallis }\end{array}$} \\
\hline & & & $A$ & $c$ & $D$ & $A$ & C & $D$ & $\mathbf{H}$ & $p$ \\
\hline \multirow{5}{*}{$\begin{array}{l}\text { Brachychthoniidae } \\
\text { Thor, } 1934\end{array}$} & Liochthonius brevis (Michael, 1888) & & 0.0 & & & 1.3 & 17 & 0.07 & 0.83 & ns \\
\hline & L. neglectus Moritz, 1976 & L.neg & 0.0 & & & 39.5 & 33 & 1.97 & 1.83 & ns \\
\hline & L. tuxeni (Forsslund, 1957) & & 0.6 & 20 & 0.07 & 0.2 & 17 & 0.01 & 0.07 & ns \\
\hline & $\begin{array}{l}\text { Neobrachychthonius magnus } \\
\text { Moritz, } 1976\end{array}$ & N.mag & 0.0 & & & 46.0 & 67 & 2.29 & 4.47 & 0.035 \\
\hline & $\frac{\text { Sellnickochthonius jacoti }}{\text { (Evans, 1952) }}$ & & 0.0 & & & 0.7 & 17 & 0.03 & 0.83 & ns \\
\hline $\begin{array}{l}\text { Eniochthoniidae } \\
\text { Grandjean, } 1947\end{array}$ & $\begin{array}{l}\text { Eniochthonius minutissimus } \\
\text { (Berlese, 1904) }\end{array}$ & & 0.2 & 20 & 0.02 & 2.2 & 17 & 0.11 & 0.00 & ns \\
\hline $\begin{array}{l}\text { Hypochthoniidae } \\
\text { Berlese, } 1910\end{array}$ & Hypochthonius rufulus C.L. Koch, 1835 & & 2.2 & 40 & 0.27 & 0.8 & 17 & 0.04 & 0.66 & ns \\
\hline \multirow{4}{*}{$\begin{array}{l}\text { Euphthiracaridae } \\
\text { Jacot, } 1930\end{array}$} & Acrotritia ardua (C.L. Koch, 1841) & A.ard & 8.6 & 100 & 1.04 & 0.0 & & & 8.97 & 0.003 \\
\hline & A. duplicata (Grandjean, 1953) & & 0.0 & & & 0.2 & 17 & 0.01 & 0.83 & ns \\
\hline & Euphthiracarus cribrarius (Berlese, 1904) & & 0.2 & 20 & 0.02 & 0.7 & 33 & 0.03 & 0.49 & ns \\
\hline & Phthiracarus anonymus Grandjean, 1933 & & 2.8 & 40 & 0.34 & 0.2 & 17 & 0.01 & 1.09 & ns \\
\hline & P. bryobius Jacot, 1930 & & 2.0 & 80 & 0.24 & 1.3 & 67 & 0.07 & 0.31 & ns \\
\hline Phthiracaridae & P. clavatus Parry, 1979 & & 0.0 & & & 1.5 & 17 & 0.07 & 0.83 & ns \\
\hline \multirow{3}{*}{ Perty, 1841} & P. crinitus (C.L. Koch, 1841) & & 0.2 & 20 & 0.02 & 0.0 & & & 1.20 & ns \\
\hline & P. laevigatus (C.L. Koch, 1841) & & 0.2 & 20 & 0.02 & 0.0 & & & 1.20 & ns \\
\hline & P. longulus (C.L. Koch, 1841) & & 0.4 & 40 & 0.05 & 3.5 & 83 & 0.17 & 3.31 & ns \\
\hline \multirow{3}{*}{$\begin{array}{l}\text { Steganacaridae } \\
\text { Niedbała, } 1986\end{array}$} & Atropacarus striculus (C.L. Koch, 1835) & A.str & 115.8 & 100 & 14.02 & 0.0 & & & 8.92 & 0.003 \\
\hline & Steganacarus magnus (Nicolet, 1855) & & 3.4 & 60 & 0.41 & 0.2 & 17 & 0.01 & 2.53 & ns \\
\hline & S. spinosus (Sellnick, 1920) & & 0.6 & 40 & 0.07 & 8.8 & 83 & 0.44 & 3.51 & ns \\
\hline \multirow{3}{*}{$\begin{array}{l}\text { Crotoniidae } \\
\text { Thorell, } 1876\end{array}$} & Camisia biurus (C.L. Koch, 1839) & & 0.0 & & & 2.0 & 33 & 0.10 & 1.83 & ns \\
\hline & C. spinifer (C.L. Koch, 1836) & & 0.0 & & & 0.2 & 17 & 0.01 & 0.83 & ns \\
\hline & Platynothrus peltifer (C.L. Koch, 1839) & P.pel & 19.0 & 80 & 2.30 & 0.2 & 17 & 0.01 & 5.24 & 0.022 \\
\hline \multirow{2}{*}{$\begin{array}{l}\text { Malaconothridae } \\
\text { Berlese, } 1916\end{array}$} & $\begin{array}{l}\text { Malaconothrus monodactylus } \\
\text { (Michael, 1888) }\end{array}$ & M.mon & 71.2 & 60 & 8.62 & 0.0 & & & 4.37 & 0.037 \\
\hline & Tyrphonothrus maior (Berlese, 1910) & T.mai & 98.6 & 100 & 11.94 & 0.00 & & & 8.92 & 0.003 \\
\hline $\begin{array}{l}\text { Nanhermanniidae } \\
\text { Sellnick, } 1928\end{array}$ & Nanhermannia coronata Berlese, 1913 & & 6.4 & 80 & 0.78 & 0.0 & & & 6.44 & 0.011 \\
\hline $\begin{array}{c}\text { Nothridae } \\
\text { Berlese, } 1896\end{array}$ & Nothrus silvestris Nicolet, 1855 & & 0.0 & & & 2.7 & 67 & 0.13 & 4.47 & 0.035 \\
\hline \multirow{3}{*}{$\begin{array}{l}\text { Damaeidae } \\
\text { Berlese, } 1896\end{array}$} & Damaeus clavipes (Hermann, 1804) & & 0.0 & & & 0.2 & 17 & 0.01 & 0.83 & ns \\
\hline & D. gracilipes (Kulczynski, 1902) & & 0.0 & & & 0.3 & 17 & 0.02 & 0.83 & ns \\
\hline & Porobelba spinosa (Sellnick, 1920) & & 0.0 & & & 8.7 & 83 & 0.43 & 6.19 & 0.013 \\
\hline $\begin{array}{l}\text { Cepheusidae } \\
\text { Berlese, } 1896\end{array}$ & Cepheus cepheiformis (Nicolet, 1855) & & 0.0 & & & 0.5 & 17 & 0.02 & 0.83 & ns \\
\hline \multirow{2}{*}{$\begin{array}{c}\text { Caleremaeidae } \\
\text { Grandjean, } 1965 \\
\text { Eremaeidae }\end{array}$} & Caleremaeus monilipes (Michael, 1882) & C.mon & 0.0 & & & 59.8 & 33 & 2.98 & 1.83 & ns \\
\hline & Eueremaeus silvestris (Forsslund, 1956) & & 0.0 & & & 1.0 & 33 & 0.05 & 1.83 & ns \\
\hline Oudemans, 1900 & E. valkanovi (Kunst, 1957) & & 0.0 & & & 18.8 & 50 & 0.94 & 3.03 & ns \\
\hline \multirow{2}{*}{$\begin{array}{l}\text { Astegistidae } \\
\text { Balogh, } 1961\end{array}$} & Cultroribula bicultrata (Berlese, 1905) & & 0.0 & & & 0.3 & 33 & 0.02 & 1.85 & ns \\
\hline & $\begin{array}{l}\text { Furcoribula furcillata } \\
\text { (Nordenskiöld, 1901) }\end{array}$ & & & & & 1.3 & 33 & 0.07 & 1.83 & ns \\
\hline Liacaridae & Adoristes ovatus (C.L. Koch, 1839) & & 0.8 & 60 & 0.10 & 4.2 & 67 & 0.21 & 0.89 & ns \\
\hline Sellnick, 1928 & Liacarus coracinus (C.L. Koch, 1841) & & 0.0 & & & 13.2 & 33 & 0.66 & 1.83 & ns \\
\hline
\end{tabular}


Table 1. Cont.

\begin{tabular}{|c|c|c|c|c|c|c|c|c|c|c|}
\hline \multirow{2}{*}{$\begin{array}{c}\begin{array}{c}\text { Order/Suborder } \\
\text { and Family }\end{array} \\
\text { Oribatida }\end{array}$} & \multirow[t]{2}{*}{ Species } & \multirow[t]{2}{*}{$\begin{array}{l}\text { Abbreviations } \\
\text { for DCA } \\
\text { Analysis }\end{array}$} & \multicolumn{3}{|c|}{$\begin{array}{c}\text { Microhabitat } \\
\text { A }\end{array}$} & \multicolumn{3}{|c|}{$\begin{array}{c}\text { Microhabitat } \\
\text { B }\end{array}$} & \multicolumn{2}{|c|}{$\begin{array}{l}\text { ANOVA Rang } \\
\text { Kruskal-Wallis }\end{array}$} \\
\hline & & & $A$ & $C$ & $D$ & $A$ & $C$ & $D$ & $\mathbf{H}$ & $p$ \\
\hline \multirow{10}{*}{$\begin{array}{l}\text { Carabodidae C.L. } \\
\text { Koch, } 1843\end{array}$} & Carabodes areolatus Berlese, 1916 & C.are & 0.2 & 20 & 0.02 & 40.5 & 83 & 2.01 & 4.53 & 0.033 \\
\hline & C. coriaceus C.L. Koch, 1835 & & 0.0 & & & 0.7 & 17 & 0.03 & 0.83 & ns \\
\hline & C. femoralis (Nicolet, 1855) & & 0.0 & & & 9.5 & 33 & 0.47 & 1.83 & ns \\
\hline & C. labyrinthicus (Michael, 1879) & C.lab & 1.0 & 60 & 0.12 & 24.8 & 100 & 1.24 & 7.57 & 0.006 \\
\hline & C. marginatus (Michael, 1884) & C.mar & 0.0 & & & 24.7 & 17 & 1.23 & 0.83 & ns \\
\hline & C. ornatus Storkán, 1925 & & 0.2 & 20 & 0.02 & 5.8 & 83 & 0.29 & 4.07 & 0.044 \\
\hline & C. rugosior Berlese, 1916 & & 0.2 & 20 & 0.02 & & & & 1.20 & ns \\
\hline & C. tenuis Forsslund, 1953 & & 0.0 & & & 3.3 & 17 & 0.17 & 0.83 & ns \\
\hline & C. willmanni Bernini, 1975 & C.wil & 0.0 & & & 37.2 & 33 & 1.85 & 1.83 & ns \\
\hline & $\begin{array}{c}\text { Odontocepheus elongatus } \\
\text { (Michael, 1879) }\end{array}$ & & 0.0 & & & 5.7 & 83 & 0.28 & 6.23 & 0.013 \\
\hline \multirow{6}{*}{$\begin{array}{l}\text { Autognetidae } \\
\text { Grandjean, } 1960\end{array}$} & Autogneta longilamellata (Michael, 1885) & & 0.0 & & & 0.2 & 17 & 0.01 & 0.83 & ns \\
\hline & $\begin{array}{l}\text { Conchogneta dalecarlica } \\
\text { (Forsslund, 1947) }\end{array}$ & & 0.0 & & & 2.2 & 67 & 0.11 & 4.50 & 0.034 \\
\hline & Dissorhina ornata (Oudemans, 1900) & & 0.0 & & & 14.3 & 100 & 0.71 & 8.25 & 0.004 \\
\hline & Graptoppia foveolata (Paoli, 1908) & G.fov & 0.0 & & & 35.8 & 50 & 1.78 & 3.03 & ns \\
\hline & $\frac{\text { Lauroppia beskidyensis }}{(\text { Niemi et Skubala, 1993) }}$ & & 0.0 & & & 0.2 & 17 & 0.01 & 0.83 & ns \\
\hline & Microppia minus (Paoli, 1908) & & 0.0 & & & 0.5 & 50 & 0.02 & 3.13 & ns \\
\hline \multirow{9}{*}{$\begin{array}{c}\text { Oppiidae } \\
\text { Sellnick, } 1937\end{array}$} & Moritzoppia keilbachi (Moritz, 1969) & M.kei & 0.0 & & & 26.8 & 83 & 1.33 & 6.19 & 0.013 \\
\hline & M. translamellata (Willmann, 1923) & M.tra & 15.6 & 20 & 1.89 & 0.0 & & & 1.20 & ns \\
\hline & Oppiella falcata (Paoli, 1908) & O.fal & 0.0 & & & 465.5 & 100 & 23.16 & 8.25 & 0.004 \\
\hline & O. neerlandica (Oudemans, 1900) & O.nee & 60.0 & 100 & 7.27 & 0.0 & & & 8.92 & 0.003 \\
\hline & O. nova (Oudemans, 1902) & O.nov & 47.6 & 100 & 5.76 & 67.7 & 100 & 3.37 & 0.21 & ns \\
\hline & $\begin{array}{l}\text { O. propinqua Mahunka et } \\
\text { Mahunka-Papp, } 2000\end{array}$ & O.pro & 38.8 & 100 & 4.70 & 0.0 & & & 8.92 & 0.003 \\
\hline & O. splendens (C.L. Koch, 1841) & O.spl & 0.0 & & & 215.2 & 83 & 10.70 & 6.19 & 0.013 \\
\hline & O. uliginosa (Willmann, 1919) & & & & & 0.7 & 33 & 0.03 & 1.83 & ns \\
\hline & $\begin{array}{l}\text { Rhinoppia subpectinata } \\
\text { (Oudemans, 1900) }\end{array}$ & R.sub & 0.0 & & & 24.0 & 50 & 1.19 & 3.03 & ns \\
\hline \multirow{6}{*}{$\begin{array}{c}\text { Quadroppiidae } \\
\text { Balogh, } 1983 \\
\text { Thyrisomidae } \\
\text { Grandjean, } 1953\end{array}$} & Quadroppia monstruosa (Hammer, 1979) & & 0.0 & & & 3.3 & 50 & 0.17 & 3.03 & ns \\
\hline & Q. quadricarinata (Michael, 1885) & Q.qua & 0.0 & & & 48.8 & 100 & 2.43 & 8.25 & 0.004 \\
\hline & Banksinoma lanceolata (Michael, 1885) & & 0.6 & 40 & 0.07 & 0.2 & 17 & 0.01 & 0.87 & ns \\
\hline & Suctobelba regia Moritz, 1970 & & 0.0 & & & 13.2 & 83 & 0.66 & 6.19 & 0.013 \\
\hline & S. trigona (Michael, 1888) & & 0.0 & & & 2.0 & 17 & 0.10 & 0.83 & ns \\
\hline & Suctobelbella lobata (Strenzke, 1950) & & 0.0 & & & 1.0 & 17 & 0.05 & 0.83 & ns \\
\hline \multirow{7}{*}{$\begin{array}{l}\text { Suctobelbidae } \\
\text { Jacot, } 1938\end{array}$} & $\frac{\text { S. carcharodon }}{\text { (Moritz, 1966) }}$ & & 0.0 & & & 0.8 & 17 & 0.04 & 0.83 & ns \\
\hline & S. falcata (Forsslund, 1941) & S.fal & 0.0 & & & 42.7 & 33 & 2.12 & 1.83 & ns \\
\hline & S. sarekensis (Forsslund, 1941) & & 0.0 & & & 18.7 & 50 & 0.93 & 3.03 & ns \\
\hline & S. similis (Forsslund, 1941) & & 0.0 & & & 0.3 & 17 & 0.02 & 0.83 & ns \\
\hline & Suctobelbella sp. 1 & & 0.0 & & & 2.7 & 17 & 0.13 & 0.83 & ns \\
\hline & S. subcornigera (Forsslund, 1941) & S.sbc & 2.6 & 60 & 0.31 & 87.8 & 100 & 4.37 & 6.13 & 0.013 \\
\hline & S. subtrigona (Oudemans, 1900) & S.sbt & 0.0 & & & 75.8 & 67 & 3.77 & 4.47 & 0.035 \\
\hline $\begin{array}{l}\text { Tectocepheidae } \\
\text { Grandjean, } 1954\end{array}$ & Tectocepheus velatus (Michael, 1880) & T.vel & 16.6 & 100 & 2.01 & 302.0 & 100 & 15.02 & 7.50 & 0.006 \\
\hline $\begin{array}{l}\text { Licneremaeidae } \\
\text { Grandjean, } 1954\end{array}$ & $\begin{array}{c}\text { Licneremaeus licnophorus } \\
\text { (Michael, 1882) }\end{array}$ & & 0.0 & & & 0.7 & 17 & 0.03 & 0.83 & ns \\
\hline Phenopelopidae & Eupelops plicatus (C.L. Koch, 1835) & & 0.2 & 20 & 0.02 & 0.7 & 50 & 0.03 & 1.15 & ns \\
\hline Petrunkevich, 1955 & E. torulosus (C.L. Koch, 1839) & & 1.0 & 60 & 0.12 & 10.5 & 100 & 0.52 & 7.11 & 0.008 \\
\hline \multirow{3}{*}{$\begin{array}{l}\text { Achipteriidae } \\
\text { Thor, } 1929\end{array}$} & Achipteria magna (Sellnick, 1928) & A.mag & 0.0 & & & 63.2 & 67 & 3.14 & 4.47 & 0.035 \\
\hline & A. nitens (Nicolet, 1855) & & 0.0 & & & 5.3 & 33 & 0.27 & 1.83 & ns \\
\hline & Parachipteria fanzagoi (Jacot, 1929) & P.fan & 278.8 & 100 & 33.76 & 1.5 & 33 & 0.07 & 7.86 & 0.005 \\
\hline \multirow{2}{*}{$\begin{array}{l}\text { Oribatellidae } \\
\text { Jacot, } 1925\end{array}$} & Oribatella quadricornuta Michael, 1880 & & 0.0 & & & 0.2 & 17 & 0.01 & 0.83 & ns \\
\hline & Ophidiotrichus tectus (Michael, 1884) & & 0.0 & & & 0.2 & 17 & 0.01 & 0.83 & ns \\
\hline
\end{tabular}


Table 1. Cont.

\begin{tabular}{|c|c|c|c|c|c|c|c|c|c|c|}
\hline \multirow{2}{*}{$\begin{array}{c}\begin{array}{c}\text { Order/Suborder } \\
\text { and Family }\end{array} \\
\text { Oribatida }\end{array}$} & \multirow[t]{2}{*}{ Species } & \multirow[t]{2}{*}{$\begin{array}{l}\text { Abbreviations } \\
\text { for DCA } \\
\text { Analysis }\end{array}$} & \multicolumn{3}{|c|}{$\begin{array}{c}\text { Microhabitat } \\
\text { A }\end{array}$} & \multicolumn{3}{|c|}{$\begin{array}{c}\text { Microhabitat } \\
\text { B }\end{array}$} & \multicolumn{2}{|c|}{$\begin{array}{l}\text { ANOVA Rang } \\
\text { Kruskal-Wallis }\end{array}$} \\
\hline & & & $A$ & $C$ & $D$ & $A$ & $C$ & $D$ & $\mathbf{H}$ & $p$ \\
\hline $\begin{array}{l}\text { Haplozetidae } \\
\text { Grandjean, } 1936\end{array}$ & Lagenobates lagenulus (Berlese, 1904) & & 0.0 & & & 0.2 & 17 & 0.01 & 0.83 & ns \\
\hline Oribatulidae & Oribatula exilis (Nicolet, 1855) & O.exi & 0.0 & & & 85.2 & 100 & 4.24 & 8.25 & 0.004 \\
\hline Thor, 1929 & O. tibialis (Nicolet, 1855) & & 0.0 & & & 2.2 & 50 & 0.11 & 3.03 & ns \\
\hline $\begin{array}{l}\text { Parakalummidae } \\
\text { Grandjean, } 1936\end{array}$ & $\begin{array}{l}\text { Neoribates aurantiacus } \\
\text { (Oudemans, 1914) }\end{array}$ & & 0.0 & & & 0.3 & 17 & 0.02 & 0.83 & ns \\
\hline & Scheloribates initialis (Berlese, 1908) & & 3.4 & 60 & 0.41 & 4.0 & 67 & 0.20 & 0.08 & ns \\
\hline Scheloribatidae & S. pallidulus (C.L. Koch, 1841) & & 0.0 & & & 0.5 & 17 & 0.02 & 0.83 & ns \\
\hline Grandjean, 1933 & Liebstadia longior (Berlese, 1908) & & 0.0 & & & 0.2 & 17 & 0.01 & 0.83 & ns \\
\hline & L. similis (Michael, 1888) & & 0.0 & & & 1.0 & 33 & 0.05 & 1.83 & ns \\
\hline Ceratozetidae & Fuscozetes fuscipes (C.L. Koch, 1844) & F.fus & 36.6 & 100 & 4.43 & 0.0 & & & 8.92 & 0.003 \\
\hline Jacot, 1925 & $\begin{array}{l}\text { Sphaerozetes orbicularis } \\
\text { (C.L. Koch, 1835) }\end{array}$ & & 0.0 & & & 0.25 & 50 & 0.12 & 3.03 & ns \\
\hline Chamobatidae & Chamobates borealis Trägårdh, 1902 & & 0.22 & 80 & 0.27 & 0.22 & 50 & 0.11 & 0.08 & ns \\
\hline Chamobatidae & C. pusillus (Berlese, 1895) & & 0.0 & & & 0.43 & 33 & 0.22 & 1.83 & ns \\
\hline Thor, 1937 & C. rastratus (Hull, 1914) & & 0.0 & & & 0.02 & 17 & 0.01 & 0.83 & ns \\
\hline $\begin{array}{l}\text { Galumnidae } \\
\text { Jacot, } 1925\end{array}$ & Pergalumna nervosa (Berlese, 1914) & & 0.0 & & & 0.03 & 17 & 0.02 & 0.83 & ns \\
\hline \multicolumn{11}{|c|}{ Mesostigmata } \\
\hline $\begin{array}{c}\text { Epicriidae } \\
\text { Berlese, } 1885\end{array}$ & Epicrius mollis (Kramer, 1876) & E.mol & 1.8 & 40 & 4.79 & 0.3 & 17 & 0.64 & 1.09 & ns \\
\hline & Prozercon kochi Sellnick, 1943 & P.koc & 2.2 & 40 & 5.85 & 1.5 & 50 & 2.89 & 0.04 & ns \\
\hline $\begin{array}{l}\text { Zerconidae } \\
\text { Berlese, } 1892\end{array}$ & $\begin{array}{l}\text { Zercon lindrothi Lundqvist et } \\
\text { Johonston, } 1986\end{array}$ & Z.lin & 0.0 & & & 1.5 & 50 & 2.89 & 3.06 & ns \\
\hline & Z. triangularis C.L. Koch, 1836 & Z.tri & 0.0 & & & 3.5 & 50 & 6.75 & 3.06 & ns \\
\hline & Z. zelawaiensis Sellnick, 1944 & Z.zel & 0.0 & & & 30.2 & 67 & 58.20 & 4.47 & 0.035 \\
\hline $\begin{array}{l}\text { Macrochelidae } \\
\text { Vitzthum, } 1930\end{array}$ & Macrocheles opacus (C.L. Koch, 1839) & & 0.2 & 20 & 0.53 & 0.0 & & & 1.20 & ns \\
\hline \multirow{11}{*}{$\begin{array}{c}\text { Parasitidae } \\
\text { Oudemans, } 1901\end{array}$} & Holoparasitus inornatus (Berlese, 1906) & H.ino & 0.0 & & & 1.2 & 50 & 2.25 & 3.03 & ns \\
\hline & $\begin{array}{l}\text { Paragamasus celticus } \\
\text { (Bhattacharyya, 1963) }\end{array}$ & P.cel & 1.6 & 60 & 4.26 & 0.0 & & & 4.40 & 0.036 \\
\hline & P. robustus (Oudemans, 1902) & P.rob & 0.4 & 20 & 1.06 & 0.5 & 50 & 0.96 & 0.41 & ns \\
\hline & P. runcatellus (Berlese, 1903) & P.run & 0.0 & & & 1.7 & 50 & 3.22 & 3.06 & ns \\
\hline & P. parrunciger (Bhattacharyya, 1963) & P.par & 16.6 & 40 & 44.15 & 0.0 & & & 2.64 & ns \\
\hline & P. lapponicus (Trägårdh, 1910) & P.lap & 5.2 & 60 & 13.83 & 5.5 & 67 & 10.61 & 0.32 & ns \\
\hline & Pergamasus crassipes (Linne, 1758) & P.cra & 2.4 & 60 & 6.38 & 0.5 & 33 & 0.96 & 1.68 & ns \\
\hline & P. septentrionalis (Oudemans, 1902) & & 0.2 & 20 & 0.53 & 0.2 & 17 & 0.32 & 0.02 & ns \\
\hline & Parasitus lunulatus (J. Muller, 1859) & & 0.2 & 20 & 0.53 & 0.0 & & & 1.20 & ns \\
\hline & $\begin{array}{c}\text { Vulgarogamasus kraepelini } \\
\text { (Berlese, 1905) }\end{array}$ & V.kra & 0.2 & 20 & 0.53 & 1.0 & 17 & 1.93 & 0.00 & ns \\
\hline & Other Parasitidae, juveniles & Paras & 2.4 & 80 & 6.38 & 1.0 & 50 & 1.93 & 0.57 & ns \\
\hline \multirow{3}{*}{$\begin{array}{c}\text { Veigaiidae } \\
\text { Oudemnas, } 1939\end{array}$} & Veigaia cerva (Kramer, 1876) & & 0.0 & & & 0.2 & 17 & 0.32 & 0.83 & ns \\
\hline & V. kochi (Trägårdh, 190 1) & & 0.2 & 20 & 0.53 & 0.2 & 17 & 0.32 & 0.02 & ns \\
\hline & V. nemorensis (C.L. Koch, 1839) & V.nem & 0.2 & 20 & 0.53 & 2.5 & 50 & 4.82 & 1.61 & ns \\
\hline Ascidae Voigts et & Asca aphidioides (Linne, 1758) & & 0.0 & & & 0.3 & 17 & 0.64 & 0.83 & ns \\
\hline Oudemans, 1905 & Cheiroseius mutilus (Berlese, 1916) & C.mut & 1.6 & 60 & 4.26 & 0.0 & & & 4.37 & 0.037 \\
\hline $\begin{array}{l}\text { Laelapidae } \\
\text { Berlese, } 1892\end{array}$ & $\begin{array}{l}\text { Pachylaelaps dubius } \\
\text { Hirschmann et Krauss, } 1965\end{array}$ & & 0.0 & & & 0.2 & 17 & 0.32 & 0.83 & ns \\
\hline $\begin{array}{l}\text { Trachytidae } \\
\text { Trägårdh, } 1938\end{array}$ & Trachytes aegrota (C.L. Koch, 1841) & T.aeg & 2.0 & 40 & 5.32 & 0.0 & & & 2.64 & ns \\
\hline $\begin{array}{l}\text { Uropodidae } \\
\text { Kramer, } 1881\end{array}$ & Uropoda misella (Berlese, 1916) & & 0.2 & 20 & 0.53 & 0.0 & & & 1.20 & ns \\
\hline
\end{tabular}


Table 2. Preference of species for one or other of the microhabitats: A-Sphagnum on pool and, B-other mosses on medium-wet forest floor nearby in a limestone forest in Southern Norway.

\begin{tabular}{|c|c|c|c|}
\hline Species & Specificity & Fidelity & $p$-Value \\
\hline \multicolumn{4}{|c|}{$\mathrm{A}$} \\
\hline A. ardua & 1.00 & 1.00 & 0.01 \\
\hline A. striculus & 1.00 & 1.00 & 0.01 \\
\hline F. fuscipes & 1.00 & 1.00 & 0.01 \\
\hline O. neerlandica & 1.00 & 1.00 & 0.01 \\
\hline O. propinqua & 1.00 & 1.00 & 0.01 \\
\hline P. fanzagoi & 0.99 & 1.00 & 0.01 \\
\hline N. coronata & 1.00 & 0.80 & 0.04 \\
\hline T. maior & 1.00 & 0.80 & 0.05 \\
\hline P. peltifer & 0.98 & 0.80 & 0.04 \\
\hline \multicolumn{4}{|c|}{ B } \\
\hline D. ornata & 1.00 & 1.00 & 0.01 \\
\hline O. falcata & 1.00 & 1.00 & 0.01 \\
\hline Q. quadricartinata & 1.00 & 1.00 & 0.01 \\
\hline O. exilis & 1.00 & 1.00 & 0.01 \\
\hline S. subcornigera & 0.97 & 1.00 & 0.02 \\
\hline C. labyrinthicus & 0.96 & 1.00 & 0.01 \\
\hline E. torulosus & 0.93 & 1.00 & 0.04 \\
\hline M. keilbachi & 1.00 & 0.83 & 0.05 \\
\hline O. elongatus & 1.00 & 0.83 & 0.02 \\
\hline O. splendens & 1.00 & 0.83 & 0.03 \\
\hline P. spinosa & 1.00 & 0.83 & 0.05 \\
\hline S. regia & 1.00 & 0.83 & 0.02 \\
\hline C. areolatus & 0.99 & 0.83 & 0.05 \\
\hline C. ornatus & 0.97 & 0.83 & 0.05 \\
\hline
\end{tabular}
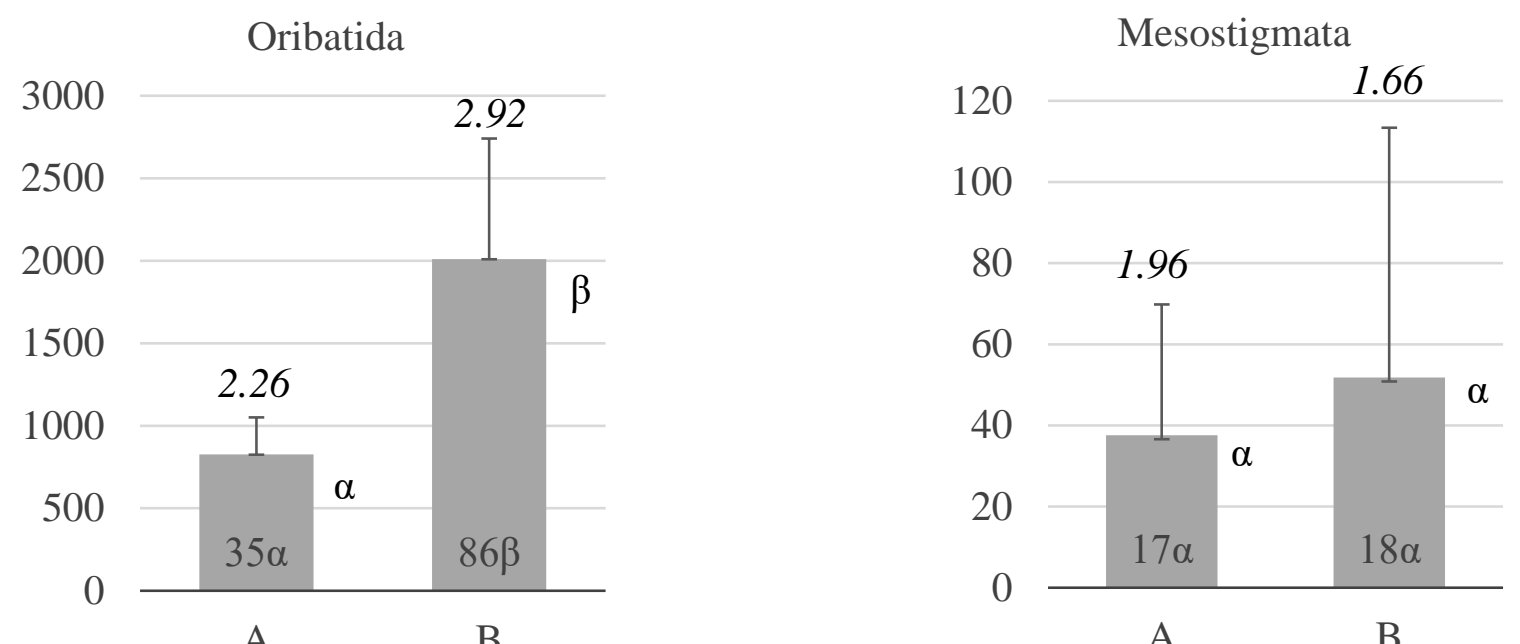

Figure 3. Average abundance (in $500 \mathrm{~cm}^{3}$ ) of mites (bars) with standard deviation (whiskers), Shannon index (above bars) and number of species (within bars) in the two microhabitats: A Sphagnum on pool and, B other mosses on medium-wet forest floor nearby in a limestone forest in Southern Norway; different Greek letters indicate significant differences at $p \leq 0.05$.

Thirty-five species of Oribatida and 17 species of Mesostigmata were found in Sphagnum mosses (Table 1). Among Oribatida the most abundant was Parachipteria fanzagoi (Jacot, 1929), which comprised over 30\% of all Oribatida collected and was followed by Atropacarus striculus (C.L. Koch, 1835) and Tyrphonothrus maior (Berlese, 1910) (which made $14 \%$ and $12 \%$ of Oribatida, respectively). These three species showed clear preferences to the Sphagnum microhabitat on the pool (Table 2). Among Mesostigmata, Paragamasus parrunciger (Bhattacharyya, 1963) dominated (it comprised 44\% of Mesostigmata), fol- 
lowed by P. lapponicus (Trägårdh, 1910) which comprised about $14 \%$ of mesotigmatid mites. None of the Mesostigmata species showed clear preferences to the microhabitat on the pool (Table 2).

In total, 86 species of Oribatida and 18 species of Mesostigmata were found in the second microhabitat. Most of the species abundant in Sphagnum, except P. lapponicus, were either absent or very uncommon here. The most abundant oribatid species was Oppiella neerlandica (Oudemans, 1900) which comprised approximately 20\% of Oribatida and was followed by Tectocepheus velatus (Michael, 1880) and Oppiella splendens (C.L. Koch, 1841) (15\% and $11 \%$ of Oribatida, respectively). Among Mesostigmata more than $55 \%$ of the individuals were Zercon zelawaiensis Sellnick, 1944 while P. lapponicus comprised approximately $11 \%$ of this group.

There were significant differences between the communities in both microhabitats as assessed by PERMANOVA (Oribatida $\mathrm{F}=9.4143, p=0.004$, Mesostigmata $\mathrm{F}=3.0248$, $p=0.009)$ and these communities were clearly grouped by detrended correspondence analysis (Figure 4). The abundance of most Oribatida species with dominance index above 1 differed significantly between the two microhabitats, while in Mesostigmata, significant differences were observed in only a few cases (Table 1).

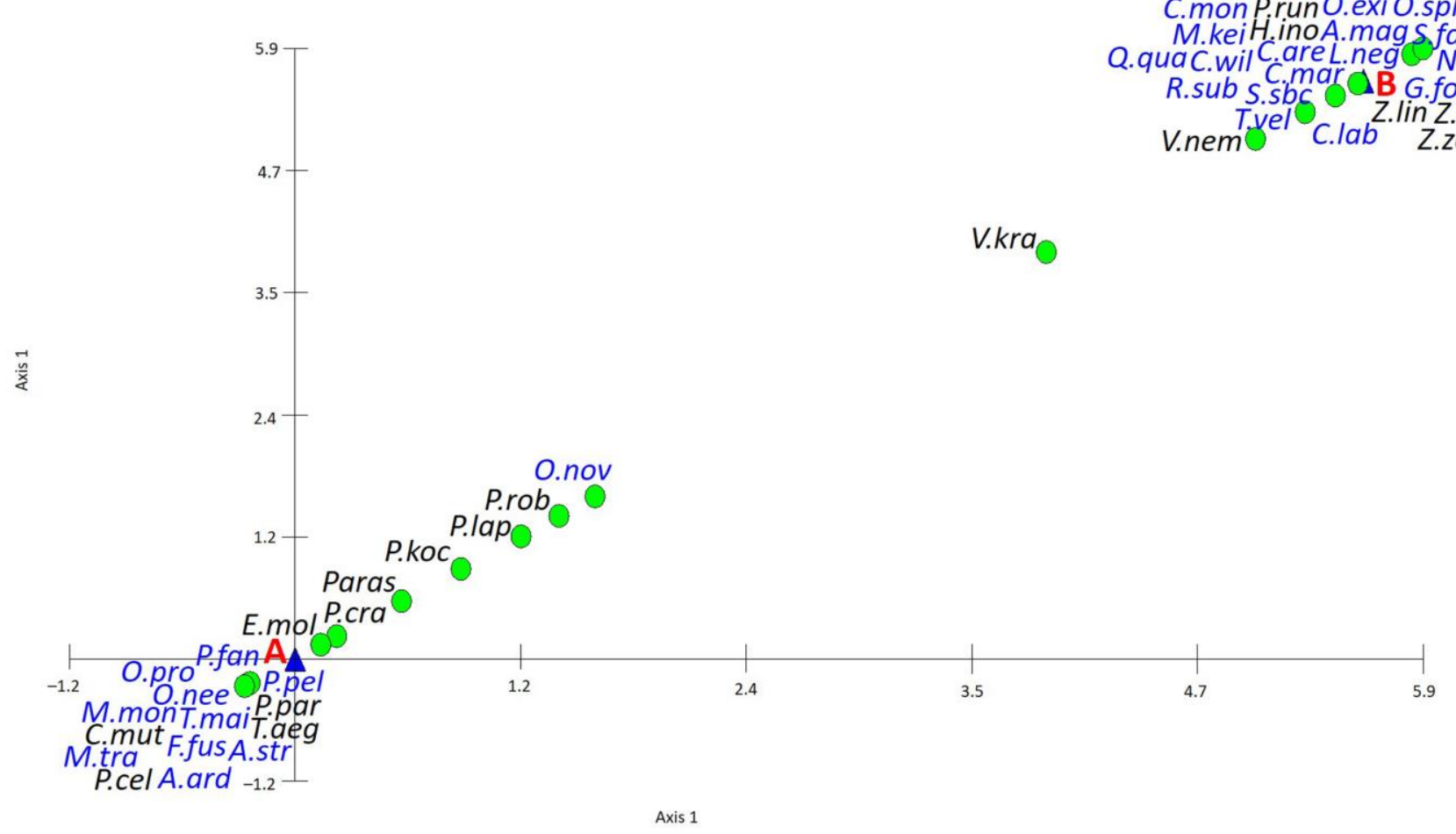

Figure 4. Detrended correspondence analysis (DCA) for species of Oribatida and Mesostigmata communities with $D \geq 1.0$ in two microhabitats: A-Sphagnum on pool and, B-other mosses on medium-wet forest floor nearby in a limestone forest in Southern Norway; eigenvalues for axis $1 \kappa=0.906(100,0 \%)$, for axis $2 \kappa=<0.001(<0.001 \%)$; triangles indicate distribution of microhabitats and circles indicate distribution of species in DCA ordination space; Oribatida are marked in blue, Mesostigmata in black; see Table 1 for abbreviations of species names.

Five species newly recorded for Norway were determined: Graptoppia foveolata (Paoli, 1908), Lauroppia beskidyensis (Niemi et Skubala, 1993), Sellnickochthonius jacoti (Evans, 1952), Suctobelbella carcharodon (Moritz, 1966) (Oribatida), and Pachylaelaps dubius Hirschmann et Krauss, 1965 (Mesostigmata) —all of them occurred only in medium-wet forest floor. The latter four species were also new records to Fennoscandia (Table 1). 


\section{Discussion}

The oribatid communities differed more between the two microhabitats than did the mesostigmatid communities. This shows that oribatid communities are more variable between microhabitats studied compared to Mesostigmata and their overall species richness is, therefore, more affected by the microhabitat diversity in the forest. Similar observations were made in studies of other microhabitats (beech litter, moss on beech litter, moss on beech stumps, rotting beech wood, damp litter, and moss on beech trunks) studied in a beech forest reserve in Poland [95]. Oribatid mites are mainly saprophagous, and therefore are more dependent on the type of vegetation, while Mesostigmata are mainly predators, feeding on nematodes, springtails, juvenile mites, and some insect larvae [96]. Therefore, they need to be more mobile, looking for their prey, whereas the slower moving Oribatida are surrounded by stationary food resources [95].

The number of oribatid species found at the pool was similar to the records from the edge of 16 water bodies in Northern Poland [4]. In Poland, with a two times higher sampling effort (10 replicates vs. 5 replicates in the present study) the average number of species was 26 and ranged from 17 to 41 [4], while in the present study 35 species of Oribatida were found. Water-saturated microhabitats are challenging for Oribatida, and most oribatid species prefer high or medium values of humidity [97,98]. Therefore, it is not surprising that Oribatida were significantly less abundant and less diverse at the pool. However, there are species, that are specifically either aquatic (i.e., with reproduction and all life stages inhabiting submerged habitats) or amphibious (i.e., living in water but seem to need saturated air to reproduce), adapted to live there [99], and therefore require wet microhabitats to live in woodlands.

Sphagnum mosses were dominated by the species characteristic of wet habitats, including Parachipteria fanzagoi, Tyrphonothrus maior, and Atropacarus striculus. These three species together made up almost $60 \%$ of all Oribatida there, while in the alternative microhabitat they were absent (T. maior, A. striculus) or very few (P. fanzagoi). The same species were also recorded abundantly in mires in Western Norway [53], while at the pond in Poland they occurred less abundantly but nevertheless were a stable component of the oribatid communities found in all seasons [10]. Among 46 Holarctic mires analyzed, P. fanzagoi was found in seven of them, T. maior in 24 and A. striculus in 29 [100].

Parachipteria fanzagoi was found in all studied microhabitats in mires in Western Norway but was particularly abundant in the Sphagnum section Acutifolia. Its age structure was similar in all compared microhabitats, and in July the juveniles made $70 \%$ of its populations [53]. In contrast, $A$. striculus did not show preferences to any Sphagnum [53]. It was represented in extracted samples only by the adults and, therefore, it is likely that juveniles are found elsewhere. Ptyctimous mites, to which A. striculus belongs to, have a remarkable ecology where immatures form galleries inside dead wood, cones or conifer needles and do not leave them before adult stage. They can only be obtained by dissecting these shelters and, therefore, are difficult to find [101,102].

Although P. fanzagoi and A. striculus occur frequently in mires, they are also found in damp forests and meadows $[7,18,25,103]$. For example, in a broadleaf forest in Eastern Norway P. fanzagoi was the second most abundant and frequent species [8]. In turn, $A$. striculus was the most abundant ptyctimous species in several broadleaf forests studied in Norway and comprised nearly $30 \%$ of all ptyctimous mites collected [11]. It was also the most common and abundant ptyctimous mite in studies in Finland [73] and in the Białowieża primeval forest in Poland [104].

In contrast to the species mentioned above, Tyrphonothrus maior is aquatic and, as the name implies, a tyrphobiont, i.e., restricted to mires. It lives in Sphagnum mosses and feeds on them [105]; in forests it can only be found in Sphagnum pools like the one studied here. Although few oribatid species are found solely in mires [106], their total diversity in undisturbed peatlands can be comparable to forests [53]. Wet Sphagnum-dominated habitats must therefore be underestimated in terms of their biodiversity value. 
Another example on how water bodies make forest diversity more unique is the mesostigmatid species Cheiroseius mutilus (Berlese, 1916), that is found only in watersoaked microhabitats. It was previously recorded from mires in Norway [80] and Finland [66]. Although the communities of Mesostigmata did not include species that were clear indicators of either microhabitat, some of the collected species are more specialized. One of them is Epicrius mollis (Kramer, 1876), which can be found in forests, but more so in mires $[107,108]$. The most abundant Mesostigmata species in this study, Paragamasus parrunciger, is mainly found in forests, meadows and pastures [108,109], but also occurs abundantly in wetlands [66]. However, Mesostigmata communities are relatively depauperate in mire habitats [109].

In addition to showing an interesting contribution of the forest pool in increasing the mites' diversity, this study also revealed a very interesting acarofauna in a very limited patch of limestone forest. A quarter of the species found had not previously been reported from Norwegian broadleaf forests, and five species were recorded as new for Norway, including four new to Fennoscandia. These were all in the medium-wet microhabitat. These unique characters of a rare acarofauna, already noticed in ptyctimous Oribatida [11], are likely explained by the specific environmental conditions created by the limestone background and specific vegetation of limestone forests [110].

Quite surprisingly most oribatid species that are new records for Norway, have previously been recorded mainly in Southern and Central Europe, often in warm habitats, and are considered rare. One of these rare species is Graptoppia foveolata [111] that has been considered to be a southern palearctic species [41]. In Fennoscandia, only two specimens have been found in Southern Sweden [112], while in our study it was rather abundant. Also, Suctobelbella carcharodon has peculiar occurrences and has been found only in south-central Europe: at a gypsum slope [113], in a gypsum cave [114] and in various broadleaf forests in Germany [111], at higher altitudes in Slovakia [115] and Northern Spain [116] and in warm mountain grasslands in the Czech Republic [117]. Similarly, Lauroppia beskidyensis has been known only from mountainous habitats in Poland [118,119] and Albania [120]. It cannot be excluded that some of these rare species extended their ranges of distribution in relation to climatic changes, but the lack of earlier data on mites from the Norwegian broadleaf forests makes such comparisons impossible. However, since these species are rarely found in other countries, their presence can probably be related to the natural character of the forest studied that was also supported by the records of red-list species from other groups [14]. These examples contribute to results from other broadleaf forests in Norway $[7,8,11]$ that indicate these forests represent a real treasure of Fennoscandian and European biodiversity [121].

Author Contributions: A.S., S.S., S.R. and B.H.J. planned the study; A.S., S.R. and P.D. carried out the fieldwork; R.G., S.S. and A.S. identified Oribatida; S.K. identified Mesostigmata; J.K. sorted mites; T.B. and R.G. carried out statistical analyses; A.S. and T.B. wrote the manuscript with support of all authors. All authors have read and agreed to the published version of the manuscript.

Funding: This study was supported by the Norwegian Taxonomy Initiative (Grants No. 35-16, 70184237 and 6-20, 70184243) and by the Polish Ministry of Science and Higher Education 'Regional Initiative of Excellence' in 2019-2022 (Grant No. 008/RID/2018/19).

Institutional Review Board Statement: Not applicable.

Data Availability Statement: The data presented in this study can be found here: https://doi.org/ 10.5061/dryad.pg4f4qrqw.

Acknowledgments: We are very grateful to Wojciech Niedbała (Adam Mickiewicz University, Poland) for identification of some ptyctimous mites, to Ladislav Miko (Charles University Prague, Czech Republic) for identification of some Damaeidae, and to anonymous reviewers for invaluable comments which highly improved the quality of this paper.

Conflicts of Interest: The authors have no conflict of interest to declare that are relevant to the content of this article. 


\section{References}

1. Waldon, B. Waloryzacja Przyrodnicza Śródleśnych Oczek Pojezierza Krajeńskiego; Banaszak, J., Tobolski, K., Eds.; Park Narodowy Bory Tucholskie: Charzykowy, Poland, 2002; pp. 289-299.

2. Spyra, A. Acidic, neutral and alkaline forest ponds as a landscape element affecting the biodiversity of freshwater snails. Sci. Nat. 2017, 104, 73. [CrossRef]

3. Namura-Ochalska, A. Śródleśne jeziora oligo-humotroficzne jako naturalne zbiorniki retencyjne. Stud. Mater. Cent. Eduk. Przyr. Leś. 2008, 2, 125-139.

4. Seniczak, A. Mites (Acari) of the Shores of Forest Lakes and Ponds in Northern Poland, with Species Analysis of Oribatida; Wydawnictwa UTP: Bydgoszcz, Poland, 2011; pp. 1-231, ISSN 0209-0597.

5. Hågvar, S.; Amundsen, T. Effects of liming and artificial acid rain on the mite (Acari) fauna in coniferous forest. Oikos 1981, 37, 7-20. [CrossRef]

6. Hågvar, S. Ecological spotlights on mites (Acari) in Norwegian conifer forests: A review. Pests Control Acarol. 2018. [CrossRef]

7. Seniczak, A.; Bolger, T.; Roth, S.; Seniczak, S.; Djursvoll, P.; Jordal, B.H. Diverse mite communities (Acari: Oribatida, Mesostigmata) from a broadleaf forest in western Norway. Ann. Zool. Fenn. 2019, 56, 121-136. [CrossRef]

8. Seniczak, A.; Seniczak, S.; Starý, J.; Kaczmarek, S.; Jordal, B.H.; Kowalski, J.; Roth, S.; Djursvoll, P.; Bolger, T. High diversity of mites (Acari: Oribatida, Mesostigmata) supports the high conservation value of a broadleaf forest in Eastern Norway. Forests 2021, 12, 1098. [CrossRef]

9. Walter, D.E.; Proctor, H.C. Mites: Ecology, Evolution and Behaviour; Springer: Dordrecht, The Netherlands; Heidelberg, Germany; New York, NY, USA; London, UK, 2013. [CrossRef]

10. Seniczak, A.; Seniczak, S.; Graczyk, R.; Waldon-Rudzionek, B.; Nowicka, A.; Pacek, S. Seasonal Dynamics of Oribatid Mites (Acari, Oribatida) in a Bog in Poland. Wetlands 2019, 39, 853-864. [CrossRef]

11. Seniczak, A.; Niedbała, W.; Iturrondobeitia, J.C.; Seniczak, S.; Roth, S.; Jordal, B.H. Type of broadleaf forest matters most for ptyctimous mite communities (Acari, Oribatida) in Norway. Biodivers. Conserv. 2021, 30, 2929-2953. [CrossRef]

12. Meteorological Institute. Available online: https://www.met.no (accessed on 8 June 2021).

13. Moen, A.; Lillethun, A. National Atlas of Norway: Vegetation; Norwegian Mapping Authorities: Hønefoss, Norway, 1999.

14. Miljødirektoratet. Available online: https:/ / faktaark.naturbase.no (accessed on 18 July 2021).

15. Ghiljarov, M.S.; Krivolutskij, D.A. Opredelitel Obitajushchikh v Pochve Kleshchei, Sarcoptiformes; Nauka: Moskva, Russia, 1975; pp. 1-492.

16. Pérez-Íñigo, C. Acari, Oribatei, Poronota. In Fauna Ibérica; Ramos, M.A., Ed.; Museo Nacional de Ciencias Naturales CSIC: Madrid, Spain, 1993; Volume 3, pp. 1-320.

17. Pérez-Íñigo, C. Acari, Oribatei, Gymnonota, I. In Fauna Ibérica; Ramos, M.A., Ed.; Museo Nacional de Ciencias Naturales CSIC: Madrid, Spain, 1997; Volume 9, pp. 1-373.

18. Weigmann, G. Hornmilben (Oribatida). In Die Tierwelt Deutschlands; Goecke and Evers: Keltern, Germany, 2006; Volume 76, pp. $1-520$.

19. Seniczak, S. The morphology of juvenile stages of moss mites of the family Pelopidae Ewing (Acarida: Oribatida), II. Ann. Zool. 1988, 41, 383-393.

20. Seniczak, S. The morphology of juvenile stages of moss-mites of the subfamily Sphaerozetinae (Acarida: Oribatida), II. Ann. Zool. 1989, 42, 237-248.

21. Seniczak, S. The morphology of juvenile stages of moss mites of the family Camisiidae (Acari: Oribatida), V. Zool. Anz. 1991, 227, 173-184.

22. Seniczak, S. The morphology of juvenile stages of moss mites of the family Malaconothridae (Acari, Oribatida). I. Zool. Anz. 1993, 231, 59-72.

23. Seniczak, S.; Klimek, A. The morphology of juvenile stages of moss mites of the family Camisiidae (Acari: Oribatida). Zool. Anz. 1990, 225, 71-86.

24. Seniczak, S.; Żelazna, E. The Morphology of juvenile stages of moss mites of the family Nothridae (Acari, Oribatida). II Zool. Anz. 1992, 229, 149-162.

25. Seniczak, S.; Seniczak, A. Morphology of juvenile stages of Parachipteria bella (Sellnick, 1928) and P. willmanni Hammen, 1952 (Acari: Oribatida: Achipteriidae). Ann. Zool. 2007, 57, 533-540. [CrossRef]

26. Seniczak, S.; Seniczak, A. Morphology of three species of Crotonioidea Thorel, 1876 (Acari: Oribatida), and relations between some genera. Zool. Anz. 2009, 248, 195-211. [CrossRef]

27. Seniczak, S.; Seniczak, A. Differentiation of external morphology of Damaeidae (Acari: Oribatida) in light of the ontogeny of three species. Zootaxa 2011, 2775, 1-36. [CrossRef]

28. Seniczak, A.; Seniczak, S. Morphological ontogeny of Caleremaeus monilipes (Michael, 1882) (Acaria: Oribatida: Caleremaeidae) with comments on Caleremaeus Berlese. Syst. Appl. Acarol. 2019, 24, 1995-2009. [CrossRef]

29. Ermilov, S.G. Ontogeny of oribatid mite Nanhermannia coronata (Acari, Oribatida, Nanhermanniidae). Zool. Zhurn. 2009, 88, 429-437. [CrossRef]

30. Ermilov, S.G.; Łochyńska, M. Morphology of juvenile stages of Epidamaeus kamaensis (Sellnick, 1925) and Porobelba spinosa (Sellnick, 1920) (Acari: Oribatida: Damaeidae). Ann. Zool. 2009, 59, 527-544. [CrossRef] 
31. Seniczak, S.; Norton, R.A.; Seniczak, A. Morphology of Eniochthonius minutissimus (Berlese, 1904) and Hypochthonius rufulus C.L. Koch, 1835 (Acari: Oribatida: Hypochthonioidea). Ann. Zool. 2009, 59, 373-386. [CrossRef]

32. Seniczak, S.; Seniczak, A.; Kaczmarek, S.; Słowikowska, M. Variability of external morphology of Eueremaeus Mihelčič, 1963 (Acari, Oribatida, Eremaeidae) in the light of ontogeny of three species. Int. J. Acarology 2014, 40, 81-108. [CrossRef]

33. Seniczak, A.; Seniczak, S.; Sgardelis, S.; Graczyk, R. Morphological ontogeny, distribution and ecology of Edwardzetes edwardsii and Sphaerozetes orbicularis (Acari, Oribatida, Ceratozetidae). Syst. Appl. Acarol. 2016, 21, 713-744. [CrossRef]

34. Seniczak, A.; Seniczak, S.; Graczyk, R.; Bukowski, G. Morphological ontogeny, ecology and some biological parameters of Achipteria magna (Acari: Oribatida: Achipteriidae). Syst. Appl. Acarol. 2017, 22, 980-992. [CrossRef]

35. Seniczak, A.; Seniczak, S.; Kaczmarek, S.; Chachaj, B. Morphological ontogeny and ecology of Adoristes ovatus (Acari: Oribatida: Liacaridae), with comments on Adoristes Hull. Syst. Appl. Acarol. 2017, 22, 2038-2056. [CrossRef]

36. Seniczak, S.; Seniczak, A.; Kaczmarek, S.; Marquardt, T. Morphological ontogeny, ecology and some biological parameters of Achipteria nitens (Acari: Oribatida: Achipteriidae). Zootaxa 2019, 4717, 030-046. [CrossRef]

37. Pfingstl, T.; Krisper, G. No difference in the juveniles of two Tectocepheus species (Acari: Oribatida, Tectocepheidae). Acarologia 2011, 51, 199-218. [CrossRef]

38. Ermilov, S.G.; Kolesnikov, V.B. Morphology of juvenile instars of Furcoribula furcillata and Zygoribatula exilis (Acari, Oribatida). Acarina 2012, 20, 48-59.

39. Schatz, H. Catalogue of oribatid mites (Acari: Oribatida) from Vorarlberg (Austria). Zootaxa 2020, 4783, 1-106. [CrossRef]

40. Norton, R.A.; Ermilov, S.G. Catalogue and historical overview of juvenile instars of oribatid mites (Acari: Oribatida). Zootaxa 2014, 3833, 1-132. [CrossRef]

41. Subías, L.S. Listado sistemático, sinonímico y biogeográfico de los Ácaros Oribátidos (Acariformes, Oribatida) del mundo (1758-2002). Graellsia 2021, 60, 3-305, unpublished electronic update, $15^{\mathrm{a}}$ actualización, 527p. [CrossRef]

42. Bhattacharyya, S.K. A revision of the British mites of the genus Pergamasus Berlese s lat. (Acari: Mesostigmata). Bull. Br. Mus. nat. Hist. Zool. 1963, 11, 133-242.

43. Ghiljarov, M.S.; Bregetova, N.G. Opredelitel Obitajushchikh v Pochve Kleshchei; Mesostigmata; Nauka: Moskva, Russia, 1977; pp. $1-718$.

44. Lundqvist, L.; Johnston, D.E. Description of Zercon lindrothi sp. n. and a redescription of Zercon colligans Berlese, 1920 (Acari, Mesostigmata: Zerconidae). Insect Syst. Evol. 1985, 16, 345-350. [CrossRef]

45. Micherdziński, W. Die Familie Parasitidae Oudemans 1901 (Acarina, Mesostigmata); PWN: Warszawa, Poland, 1969 ; pp. 1-690.

46. Błaszak, C. Zerconidae (Acari, Mesostigmata) Polski. Monogr. Fauny Pol. 1974, 3, 1-315.

47. Hyatt, K.H.; Emberson, R.M. A review of the Macrochelidae (Acari: Mesostigmata) of the British Isles. Bull. Br. Mus. Nat. Hist. Zool. 1988, 54, 63-125.

48. Karg, W. Acari (Acarina), Milben, Unterordnung Parasitiformes (Anactinochaeta), Uropodina Kramer, Schildkrötenmilben; Gustav Fischer Verlag: Jena, Germany, 1989; pp. 1-203.

49. Karg, W. Raubmilben: Acari (Acarina), Milben Parasitiformes (Allactinochaeta) Cohors Gamasina Leach; Gustav Fischer Verlag: Jena, Germany, 1993; pp. 1-524.

50. Gwiazdowicz, D.J. Ascid Mites (Acari, Mesostigmata) from Selected Forest Ecosystems and Microhabitats in Poland; Wydawnictwo Akademii Rolniczej im. Augusta Cieszkowskiego: Poznań, Poland, 2007; pp. 1-248.

51. Witaliński, W. Key to the world species of Holoparasitus Oudemans, 1936 (Acari: Parasitiformes: Parasitidae). Zootaxa 2017, 4277, 301-351. [CrossRef]

52. Mehl, R. Checklist of Norwegian ticks and mites (Acari). Fauna Norv. B 1979, 26, 31-45.

53. Seniczak, A.; Seniczak, S.; Iturrondobeitia, J.C.; Solhøy, T.; Flatberg, K.I. Diverse Sphagnum mosses support rich moss mite communities (Acari, Oribatida) in mires of western Norway. Wetlands 2020, 40, 1339-1351. [CrossRef]

54. Seniczak, A.; Seniczak, S.; Schwarzfeld, M.D.; Coulson, S.J.; Gwiazdowicz, D.J. Diversity and Distribution of Mites (Acari: Ixodida, Mesostigmata, Trombidiformes, Sarcoptiformes) in the Svalbard Archipelago. Diversity 2020, 12, 323. [CrossRef]

55. Seniczak, A.; Seniczak, S. Diversity of oribatid mites (Acari: Oribatida) in the Svalbard archipelago: A historical overview. Zootaxa 2020, 4834, 41-65. [CrossRef] [PubMed]

56. Seniczak, A.; Seniczak, S. Morphological ontogeny of Fuscozetes coulsoni sp. nov. (Acari: Oribatida: Ceratozetidae) from Svalbard, Norway. Syst. Appl. Acarol. 2020, 25, 680-696. [CrossRef]

57. Seniczak, A.; Seniczak, S. Morphological ontogeny of Limnozetes solhoyorum sp. nov. (Acari: Oribatida: Limnozetidae) from Norway, with comments on Limnozetes Hull. Syst. Appl. Acarol. 2020, 25, 327-348. [CrossRef]

58. Seniczak, A.; Seniczak, S. Morphological ontogeny of Limnozetes schatzi sp. nov. (Acari: Oribatida: Limnozetidae) from Norway. Syst. Appl. Acarol. 2021, 26, 1974-1991. [CrossRef]

59. Lebedeva, N.V.; Taskaeva, A.A.; Melekhina, E.N.; Zazovskaya, E.P. Invertebrates in the gradient of different age soils under a birdcliff at the Grønfjord Area (Svalbard, Arctic). Cauc. Ent. Bull. 2021, 16, 283-294. [CrossRef]

60. Thunes, K.H.; Søli, G.E.E.; Thuróczy, C.; Fjellberg, A.; Olberg, S.; Roth, S.; Coulianos, C.-C.; Disney, R.H.L.; Starý, J.; Vierbergen, G.; et al. The Arthropod Fauna of Oak (Quercus spp., Fagaceae) Canopies in Norway. Diversity 2021, $13,332$. [CrossRef]

61. Lundqvist, L. Bibliografi och checklist över Sveriges oribatider (Acari: Oribatei). Entomol. Tidskr. 1987, 108, 3-12.

62. Niemi, R.; Karppinen, E.; Uusitalo, M. Catalogue of the Oribatida (Acari) of Finland. Acta Zool. Fenn. 1997, $207,1-39$. 
63. Koponen, S.; Rinne, V.; Clayhills, T. Arthropods on oak branches in SW Finland, collected by a new trap type. Entomol. Fenn. 1997, 8, 177-183. [CrossRef]

64. Huhta, V.; Sulkava, P.; Viberg, K. Interactions between enchytraeid (Cognettia sphagnetorum), microarthropod and nematode populations in forest soil at different moistures. Appl. Soil Ecol. 1998, 9, 53-58. [CrossRef]

65. Huhta, V.; Räty, M.; Ahlroth, P.; Hänninen, S.M.; Mattila, J.; Penttinen, R.; Rintala, T. Soil fauna of deciduous forests in central Finland. Memo. Soc. Fauna Flora Fenn. 2005, 81, 52-70.

66. Huhta, V.; Siira-Pietikäinen, A.; Penttinen, R.; Räty, M. Soil fauna of Finland: Acarina, Collembola and Enchytraeidae. Memo. Soc. Fauna Flora Fenn. 2010, 86, 59-82.

67. Huhta, V.; Siira-Pietikäinen, A.; Penttinen, R. Importance of dead wood for soil mite (Acarina) communities in boreal old-growth forests. Soil Org. 2012, 84, 499-512.

68. Huhta, V.; Penttinen, R.; Pitkänen, E. Cultural factors in the distribution of soil mites in Finland. Memo. Soc. Fauna Flora Fenn. 2012, 88, 52-70.

69. Froberg, L.; Solhøy, T.; Baur, A.; Baur, B. Lichen specificity of Oribatid mites (Acari; Oribatida) on limestone walls in the Great Alvar of Gland, Sweden. Entomol. Tidskr. 2003, 124, 177-182.

70. Huhta, V.; Niemi, R. Communities of soil mites (Acarina) in planted birch stands as compared with natural forests in central Finland. Can. J. For. Res. 2003, 33, 171-180. [CrossRef]

71. Penttinen, R.; Siira-Pietikäinen, A.; Huhta, V. Oribatid mites in eleven different habitats in Finland. In Integrative Acarology: Proceedings of the 6 th European Congress of the EURAAC; Bertrand, M., Kreiter, S., McCoy, K.D., Migeon, A., Navajas, M., Tixier, M.S., Vial, L., Eds.; European Association of Acarologists: Bari, Italy, 2008; pp. 237-244.

72. Siira-Pietikäinen, A.; Penttinen, R.; Huhta, V. Oribatid mites (Acari: Oribatida) in boreal forest floor and decaying wood. Pedobiologia 2008, 52, 111-118. [CrossRef]

73. Penttinen, R.; Huhta, V. Ptyctima (Acari, Oribatida) in various habitats in Finland. In Trends in Acarology: Proceedings of the 12th International Congress on Acarology; Sabelis, M.W., Bruin, J., Eds.; Springer: Berlin/Heidelberg, Germany, 2009 ; pp. $167-170$.

74. Elo, R.A.; Penttinen, R.; Sorvari, J. Distribution of oribatid mites is moisture-related within red wood ant Formica polyctena nest mounds. Appl. Soil Ecol. 2018, 124, 203-210. [CrossRef]

75. Elo, R.A.; Penttinen, R.; Sorvari, J. A comparative study of oribatid mite communities in red wood ant Formica polyctena nests and surrounding soil in a Finnish oak forest. Insect Conserv. Divers. 2016, 9, 210-223. [CrossRef]

76. Niedbała, W.; Liu, D. Catalogue of ptyctimous mites (Acari, Oribatida) of the world. Zootaxa 2018, 4393, 1-238. [CrossRef] [PubMed]

77. Gwiazdowicz, D.J.; Gulvik, M.E. Checklist of Norwegian mesostigmatid mites (Acari, Mesostigmata). Nor. J. Entomol. 2005, 52, 117-125.

78. Gwiazdowicz, D.J.; Gulvik, M.E. The first records of five mite species (Acari, Mesostigmata) in Norway. Nor. J. Entomol. 2007, 54, 125-127.

79. Słomian, S.; Gulvik, M.E.; Madej, G.; Austad, I. Gamasina and Microgyniina (Acari, Gamasida) from soil and tree hollows at two traditional farms in Sogn og Fjordane, Norway. Nor. J. Entomol. 2005, 52, 39-48.

80. Gwiazdowicz, D.J.; Solhøy, T.; Kaasa, K. Five mesostigmatid mites (Acari, Mesostigmata) new to the Norwegian fauna. Nor. J. Entomol. 2013, 60, 8-10.

81. Bolger, T.; Devlin, M.; Seniczak, A. First records of ten species of Mesostigmata (Acari, Mesostigmata) added to the published Norwegian species list. Nor. J. Entomol. 2018, 65, 94-100.

82. Kaczmarek, S.; Marquardt, T.; Seniczak, A. A new species of Zercon (Parasitiformes: Mesostigmata) from Norway, with notes on sexual dimorphism in Zerconidae. Syst. Appl. Acarol. 2021, 26, 1676-1702. [CrossRef]

83. Huhta, V. Catalogue of the Mesostigmata mites in Finland. Memo. Soc. Fauna Flora Fenn. 2016, 92, 129-148.

84. SLU Artdatabanken. Available online: https:/ /artfakta.se (accessed on 20 September 2021).

85. Odum, E.P. Podstawy Ekologii; PWRiL: Warszawa, Poland, 1982.

86. Stanisz, A. Easy Course of Statistic Using Statistica PL and Medicine Examples, 1. Basic Statistic; StatSoft Polska: Kraków, Poland, 2006.

87. Legendre, P.; Legendre, L. Numerical Ecology, 2nd ed.; Elsevier Science BV: Amsterdam, The Netherlands, $1998 ;$ p. 853.

88. Leps, J.; Šmilauer, P. Multivariate Analysis of Ecological Data Using CANOCO; Cambridge University Press: Cambridge, UK, 2003; p. 269.

89. Dell. STATISTICA 13.3; Dell: Round Rock, TX, USA, 2020.

90. Kovach Computing Services. MVSP: A Multivariate Statistical Package for Windows, Ver. 3.0; Kovach Computing Services: Wales, UK, 2011.

91. Microsoft. MS Excel 365 Software; Microsoft: Redmond, WA, USA, 2020.

92. Anderson, M.J. A new method for non-parametric multivariate analysis of variance. Austral Ecol. 2001, $26,32-46$.

93. Dufrêne, M.; Legendre, P. Species assemblages and indicator species: The need for a flexible asymmetrical approach. Ecol. Monogr. 1997, 67, 345-366. [CrossRef]

94. De Cáceres, M.; Legendre, P. Associations between species and groups of sites: Indices and statistical inference. Ecology 2009, 90, 3566-3574. [CrossRef] 
95. Seniczak, S.; Graczyk, R.; Seniczak, A.; Faleńczyk-Koziróg, K.; Kaczmarek, S.; Marquardt, T. Microhabitat preferences of Oribatida and Mesostigmata (Acari) inhabiting lowland beech forest in Poland and the trophic interactions between these mites. Eur. J. Soil Biol. 2018, 87, 25-32. [CrossRef]

96. Harris, J.R.W.; Usher, M.B. Laboratory studies of predation by the grassland mite Pergamasus longicornis, Berlese and their possible implications for the dynamics of populations of Collembola. Sci. Proc. R. Dublin Soc. A 1976, 6, 143-153.

97. Rajski, A. Faunistic-ecological investigations on moss mites (Acari, Oribatei) in several plant associations. I. Ecol. Bull. Soc. Amis. Sc. Lettr. Poznań Ser. B 1961, 25, 123-283.

98. Niedbała, W. Mechowce-Roztocze Ekosystemów Ląowych; PWN: Warszawa, Poland, 1980; pp. 1-255.

99. Schatz, H.; Behan-Pelletier, V.M. Global diversity of oribatids (Oribatida; Acari-Arachnida). Hydrobiologia 2008, 595, 323-328. [CrossRef]

100. Mumladze, L.; Murvanidze, M.; Behan-Pelletier, V. Compositional patterns in Holarctic peat bog inhabiting oribatid mite (Acari: Oribatida) communities. Pedobiologia 2013, 56, 41-48. [CrossRef]

101. Niedbała, W. Phthiracaroidea (Acari, Oribatida): Systematic Studies; Państwowe Wydawnictwo Naukowe: Warszawa, Poland, 1992.

102. Hågvar, S. Mites (Acari) developing inside decomposing spruce needles: Biology and effect on decomposition rate. Pedobiologia 1998, 42, 358-377.

103. Weigmann, G. Oribatid communities in transects from bogs to forests in Berlin indicating the biotope qualities. In Modern Acarology; Dusbabek, F., Bukva, V., Eds.; Academia: Prague, Czech Republic, 1991; Volume 1, pp. 359-364.

104. Niedbała, W.; Błoszyk, J.; Gutowski, J.M.; Konwerski, S.; Napierała, A. A characteristic of a community of ptyctimous mites (Acari: Oribatida) in the Białowieża Primeval Forest, Central Europe. In Mites (Acari) of the Białowieża Primeval Forest; Błoszyk, J., Napierała, A., Eds.; Wydawnictwo Kontekst: Poznan, Poland, 2020; pp. 61-87.

105. Lehmitz, R.; Maraun, M. Small-scale spatial heterogeneity of stable isotopes signatures ( $\delta 15 \mathrm{~N}, \delta 13 \mathrm{C})$ in Sphagnum sp. transfers to all trophic levels in oribatid mites. Soil Biol. Biochem. 2016, 100, 242-251. [CrossRef]

106. Behan-Pelletier, V.M.; Bisset, B. Oribatida of Canadian peatlands. Mem. Entomol. Soc. Can. 1994, 126, 73-88. [CrossRef]

107. Salmane, I.; Brumelis, G. Species list and habitat preference of Mesostigmata mites (Acari, Parasitiformes) in Latvia. Acarologia 2010, 50, 373-394. [CrossRef]

108. Bolger, T.; Arroyo, J.; Piotrowska, K. A catalogue of the species of Mesostigmata (Arachnida, Acari, Parasitiformes) recorded from Ireland including information on their geographical distribution and habitats. Zootaxa 2018, 4519, 1-220. [CrossRef] [PubMed]

109. Wisdom, R.; Arroyo, J.; Bolger, T. A survey of the Oribatida and Mesostigmata (Acarina) of Irish peatlands. Bull. Irish Biogeogr. Soc. 2011, 35, 130-149.

110. Heinrichs, S.; Winterhoff, W.; Schmidt, W. Vegetation dynamics of beech forests on limestone in central Germany over half a century-Effects of climate change, forest management, eutrophication or game browsing? Biodivers. Ecol. 2012, 4, 49-61. [CrossRef]

111. Weigmann, G.; Horak, F.; Franke, K.; Christian, A. Verbreitung und Ökologie der Hornmilben (Oribatida) in Deutschland; Senckenberg Museum Für Naturkunde Görlitz: Görlitz, Germany, 2015; Volume 10, pp. 1-171.

112. Dalenius, P. The oribatid fauna of South Sweden with remarks concerning its ecology and zoogeography. Kgl. Fysiogr. Sällsk. Lund. Förh. 1950, 20, 30-48.

113. Moritz, M. Neue Oribatiden (Acari) aus Deutschland III: Suctobelba carcharodon n. sp. und Suctobelba prominens n. sp. Zool. Anz. 1966, 177, 276-282.

114. Moritz, M.; Broen, B.V.; Messner, B.; Mohrig, W. Beiträge zur Arthropodenfauna aus Großhöhlen des Harzes und des Kyffhäusers. V. Oribatei (Acarina). Deutsche Entomol. Z.N.F. 1971, 18, 133-144. [CrossRef]

115. Miko, L. Faunistic records of Slovak oribatids II. Oribatid mites (Acarina) of Busov Mountains, North-East Slovakia. Folia Faun. Slov. 2016, 21, 217-220.

116. Subías, L.S. Los ácaros oribátidos (Acari, Oribatida) de la Sierra de Albarracín (noreste de España). Rev. Ibérica Aracnol. 2015, 26, 81-84.

117. Starý, J. Půdní roztoči-pancířníci (Oribatida)—Soil mites—oribatid mites. In Grasslands of the White Carpatian Mountains; Jongepierova, I., Ed.; ZO CSOP Bílé Karpaty: Veselí nad Moravau, Czech Republic, 2008; pp. 210-212.

118. Niemi, R.; Skubała, P. New species of Moritzoppia and Medioppia from the Beskidy Mountains, Poland (Acarina, Oribatida, Oppiidae). Entomol. Fenn. 1993, 4, 195-200. [CrossRef]

119. Skubała, P.; Gurgul, B. Importance of tree hollows for biodiversity of mites (Acari) in the forest reserve "Śrubita" (Carpathian Mountains, south Poland). Biol. Lett. 2011, 48, 97-106. [CrossRef]

120. Mahunka, S.; Mahunka-Papp, L. Faunistical and taxonomical studies on oribatids collected in Albania (Acari: Oribatida), I. Opusc. Zool. Budapest 2006, 37, 43-62.

121. Håpnes, A. Background Note: Natural Forest Heritage in Norway. WWF Norway. 2003. Available online: http:/ /wwf.panda. org/wwf_news/?6748/Background-paper-Natural-forest-heritage-in-Norway (accessed on 8 June 2021). 